\title{
An Efficient Iterative Method for Computing Deflections of Bernoulli-Euler-von Karman Beams on a Nonlinear Elastic Foundation
}

\author{
Fayyaz Ahmad $d^{\mathrm{a}, \mathrm{b}, \mathrm{f}}$, Taek Soo Jang ${ }^{\mathrm{c}, *}$, Juan A. Carrasco ${ }^{\mathrm{d}}$, Shafiq Ur Rehman ${ }^{\mathrm{e}}$, Zulfiqar Ali ${ }^{\mathrm{f}}$, Nukhaze Ali $^{\mathrm{f}}$ \\ ${ }^{a}$ Dipartimento di Scienza e Alta Tecnologia, Universita dell'Insubria, Via Valleggio 11, Como 22100, Italy. \\ ${ }^{b}$ Departament de Física i Enginyeria Nuclear, Universitat Politècnica de Catalunya, Eduard Maristany 10, Barcelona 08019, Spain. \\ ${ }^{c}$ Department of Naval Architecture and Ocean Engineering, Pusan National University, Korea. \\ ${ }^{d}$ Departament d'Enginyeria Electrònica, Universitat Politècnica de Catalunya Diagonal 647, plta. 9, 08028 Barcelona, Spain. \\ ${ }^{e}$ Departmment of Mathematics, University of Engineering and Technology, Lahore 5480, Pakistan \\ ${ }^{f}$ Departament of Mathematics, Riphah International University, Faisalabad, Pakistan.
}

\begin{abstract}
An efficient iterative method is developed for the static analysis of large deflections of an infinite beam with variable cross-section resting on a nonlinear foundation. A pseudo spring constant is added and explicit matrix operators are introduced to perform differentiation through Green's function. The nonlinearity of the problem is handled with quasilinearization. To compute the solution of the quasilinear differential equation with prescribed accuracy, a new discretization method for solving quasilinear differential equations involving up to the 4th order derivative is used. The discretization method is based on relating discretizations of up to the fourth order derivative of the solution with a discretization of the solution by using a suitable Green function. Numerical experiments show that the error incurred by the discretization can be made small for the two first derivatives and that the method proposed in the paper converges fast and has good accuracy.
\end{abstract}

Keywords: Infinite beam, Variable cross-section, Nonlinear foundation, Quasilinearization, Discretization, Green's function

\section{Introduction}

The static and dynamic analysis of deflections in an infinite beam resting on a nonlinear elastic foundation find applications to the design of roads, highways and railways. Many researchers have proposed methods for static and dynamic analysis of deflections for the case of a linear elastic foundation [1, 2, 3]. Kuo and Lee [4] has performed the static analysis of deflections in an elastically restrained non uniform beam resting on a nonlinear elastic foundation using perturbation methods. In [5], the static analysis of deflections in an infinite beam resting on a nonlinear elastic foundation has been done using finite element methods. Jang et al. [6] have solved the same problem by using an iterative method. Sharma and Das Gupta [7] has performed the static analysis of an infinite beam resting on a nonlinear Winkle-type elastic foundation by using the Green's function method. The dynamic behavior of a shear deformable beam on a tensionless nonlinear viscoelastic foundation has been done in [8].

To handle deflections of an infinite beam comparable in height with the width of the beam, the nonlinear von Karman term [9]

$$
-\frac{3}{2} E A\left(\frac{d u}{d x}\right)^{2} \frac{d^{2} u}{d x^{2}}
$$

is typically included (see, for instance, [10]) in the nonlinear static fourth order Bernouilli-Euler differential equation, thus yielding the well-known Bernouilli-Euler-von Karman differential equation. Jang et al. [6] has solved the

\footnotetext{
${ }^{*}$ Corresponding author

Email address: taek@pusan.ac.kr (Taek Soo Jang)
} 
nonlinear Bernouilli-Euler differential equation by using a convergent fixed-point iterative method based on Green's function. Similar work can be found in [6, 11]. Jang [12] has also proposed a fixed-point iterative method for solving the differential equation modeling a uniform cross-section infinite beam with large deflections resting on a linear elastic foundation including the von Karman term. In that work, Jang constructed an integral equation approximating the fourth-order derivative of the deflection by using Green's function and computed lower order derivatives by differentiation of that integral equation.

Here, we consider the problem of computing the deflections of a non-uniform infinite beam with variable cross section resting on a nonlinear elastic foundation under the presence of localized external load. The linear and nonlinear coefficients modeling the nonlinear elastic foundation are allowed to be functions of the spatial variable. The nonlinearity of the problem considered is a result of both the modeling of the nonlinear elastic foundation and the von Karman term necessary to model large deflections. Whereas the nonlinearity of the differential equation is handled by using the quasilinear method (QLM). The QLM method is a generalization of the Newton-Raphson method for boundary value problems (BVPs). It was introduced by Bellman and Kalaba [13]. Mandelzweig and coworkers [14-16] proved that QLM applied to nonlinear BVPs has second order of convergence. Higher order QLM methods for specific BVPs and coupled BVPs have been proposed in [17, 18]. The convergence of QLM methods requires the initial guess to satisfy the boundary conditions and to lie in the vicinity of the solution [19]. In [6], Jang et al. converted the differential operator associated with the differential equation of an infinite beam resting on a nonlinear elastic foundation into an integral operator by introducing a pseudo spring constant, then, discretized the integral equation and used a fixed-point iterative method to solve the resulting system of algebraic equations. However, the use of the integral operator led to implicit formulations for the derivatives, making the computations expensive since solutions of nonlinear systems are required. In this paper, we consider the problem of solving the differential equation governing the static deflections of an infinite nonlinear Bernoulli-Euler-von Karman beam with variable cross section resting on a nonlinear elastic foundation under the presence of a localized external load, and develop a new method for solving that differential equation. Our method combines QLM with the discretization of the resulting quasilinear differential equation. Our discretization is such that we avoid having to solve systems of nonlinear equations, thus potentially reducing significantly the computational cost in relation to the methods proposed in [6].

The rest of the paper is organized as follows. In Section 2, we present the differential equation governing the static deflections of an infinite nonlinear Bernoulli-Euler-von Karman beam with variable cross section resting on a nonlinear elastic foundation, stating the boundary conditions that can be assumed under the presence of a localized external load. In the following, we will call that differential equation the BEVKNV differential equation. Section 3 presents the main controbution of the paper, we review QLM applied to the BEVKNV differential equation. Section 3 presents the main contribution of the paper, i.e., a discretization method for solving quasilinear differential equations involving up to the 4th order derivative under the boundary conditions of the BEVKNV differential equation. Section 5 tests the accuracy of the discretization of the derivatives in which the discretization method is based by using a function satisfying the boundary conditions. Section 6 tests experimentally the convergence and accuracy of the method for solving the BEVKNV differential equation obtained by combining QLM with the discretization method. Finally, Section 7 presents the conclusions and highlights future work. The Appendix includes a technical result used in Section 3 .

\section{Nonlinear Bernoulli-Euler-von Karman beams on a nonlinear elastic foundation with variable cross-section}

The theory of linear Bernoulli-Euler beams on a nonlinear foundation is only valid for small deflections and rotations. For moderate and large deflections and rotations, the theory has to be extended by introducing the von Karman term and is only valid provided that the strain is small. The resulting differential equation is

$$
\frac{d^{2}}{d x^{2}}\left(E(x) I(x) \frac{d^{2} u}{d x^{2}}\right)-\frac{3}{2} E(x) A(x)\left(\frac{d u}{d x}\right)^{2} \frac{d^{2} u}{d x^{2}}+f(x, u)=w(x), \quad x \in(-\infty,+\infty),
$$

where $x$ is the longitudinal coordinate, $u(x)$ is the deflection, and $E(x), I(x), A(x), f(x, u)$, and $w(x)$ are, respectively, the Young's modulus, the mass moment of inertia, the cross-section area, the law for nonlinear elastic foundation, and the applied load. Under the assumption of a localized external load, i.e. a load which vanishes outside a finite interval, 
we have the boundary conditions

$$
u, \frac{d u}{d x}, \frac{d^{2} u}{d x^{2}}, \frac{d^{3} u}{d x^{3}} \rightarrow 0 \quad \text { for } \quad|x| \rightarrow \infty .
$$

Let $S_{1}(x)=E(x) I(x)$ and $S_{2}(x)=-\frac{3}{2} E(x) A(x)$. Then, equation (1) can be rewritten as

$$
\frac{d^{2}}{d x^{2}}\left(S_{1}(x) \frac{d^{2} u}{d x^{2}}\right)+S_{2}(x)\left(\frac{d u}{d x}\right)^{2} \frac{d^{2} u}{d x^{2}}+f(x, u)=w(x) .
$$

In QLM, we obtain a sequence of approximated solutions $u_{n}(x), n=0,1,2, \ldots$. Given an approximated solution $u_{n}(x)$, the next approximated solution is written as $u_{n+1}(x)=u_{n}(x)+\delta(x)$ and a quasilinear differential equation whose solution is $u_{n+1}(x)$ is obtained. Solution of those quasilinear differential equations till convergence gives the solution of the original differential equation.

Let us derive the quasilinear differential equation on $u_{n+1}(x)$. Substituting $u(x)$ by $u_{n+1}(x)$ in (3), we obtain

$$
\frac{d^{2}}{d x^{2}}\left(S_{1}(x) \frac{d^{2}\left(u_{n}+\delta\right)}{d x^{2}}\right)+S_{2}(x)\left(\frac{d\left(u_{n}+\delta\right)}{d x}\right)^{2} \frac{d^{2}\left(u_{n}+\delta\right)}{d x^{2}}+f\left(x, u_{n}+\delta\right)=w(x) .
$$

Expanding derivatives and using a first order Taylor approximation for approximating $f\left(x, u_{n}+\delta\right)$, we obtain

$$
\begin{aligned}
& \frac{d^{2}}{d x^{2}}\left(S_{1}(x) \frac{d^{2} u_{n}}{d x^{2}}\right)+\frac{d^{2}}{d x^{2}}\left(S_{1}(x) \frac{d^{2} \delta}{d x^{2}}\right)+S_{2}(x)\left(\left(\frac{d u_{n}}{d x}\right)^{2}+\left(\frac{d \delta}{d x}\right)^{2}+2 \frac{d u_{n}}{d x} \frac{d \delta}{d x}\right)\left(\frac{d^{2} u_{n}}{d x^{2}}+\frac{d^{2} \delta}{d x^{2}}\right) \\
& +f\left(x, u_{n}\right)+f^{\prime}\left(x, u_{n}\right) \delta+O\left(\delta^{2}\right)=w(x) .
\end{aligned}
$$

Neglecting $O\left(\delta^{2}\right)$ and second and higher order terms in derivatives of $\delta(x)$, we get

$$
\begin{aligned}
& \frac{d^{2}}{d x^{2}}\left(S_{1}(x) \frac{d^{2} \delta}{d x^{2}}\right)+S_{2}(x)\left(\frac{d u_{n}}{d x}\right)^{2} \frac{d^{2} \delta}{d x^{2}}+2 S_{2}(x) \frac{d u_{n}}{d x} \frac{d^{2} u_{n}}{d x^{2}} \frac{d \delta}{d x}+f^{\prime}\left(x, u_{n}\right) \delta \\
& =-\left(\frac{d^{2}}{d x^{2}}\left(S_{1}(x) \frac{d^{2} u_{n}}{d x^{2}}\right)+S_{2}(x)\left(\frac{d u_{n}}{d x}\right)^{2} \frac{d^{2} u_{n}}{d x^{2}}+f\left(x, u_{n}\right)-w(x)\right)
\end{aligned}
$$

By using the fact that

$$
\frac{d^{2}}{d x^{2}}\left(S_{1}(x) \frac{d^{2} u_{n}}{d x^{2}}\right)+S_{2}(x)\left(\frac{d u_{n}}{d x}\right)^{2} \frac{d^{2} u_{n}}{d x^{2}}+f\left(x, u_{n}\right)-w(x) \approx 0,
$$

because $u_{n}(x)$ is approximately a solution of the original differential equation, we can approximately write (4) as

$$
\frac{d^{2}}{d x^{2}}\left(S_{1}(x) \frac{d^{2} \delta}{d x^{2}}\right)+S_{2}(x)\left(\frac{d u_{n}}{d x}\right)^{2} \frac{d^{2} \delta}{d x^{2}}+2 S_{2}(x) \frac{d u_{n}}{d x} \frac{d^{2} u_{n}}{d x^{2}} \frac{d \delta}{d x}+f^{\prime}\left(x, u_{n}\right) \delta=0 .
$$

By letting the linear differential operator

$$
L \equiv \frac{d^{2}}{d x^{2}}\left(S_{1}(x) \frac{d^{2}}{d x^{2}}\right)+S_{2}(x)\left(\frac{d u_{n}}{d x}\right)^{2} \frac{d^{2}}{d x^{2}}+2 S_{2}(x) \frac{d u_{n}}{d x} \frac{d^{2} u_{n}}{d x^{2}} \frac{d}{d x}+f^{\prime}\left(x, u_{n}\right),
$$

we can write (6) as

$$
L \delta=0 \text {. }
$$

Since $u_{n+1}(x)=u_{n}(x)+\delta(x)$, we have $L u_{n+1}=L u_{n}$. Expansion of $L u_{n+1}$ and $L u_{n}$ gives

$$
\begin{aligned}
& \frac{d^{2}}{d x^{2}}\left(S_{1}(x) \frac{d^{2} u_{n+1}}{d x^{2}}\right)+S_{2}(x)\left(\frac{d u_{n}}{d x}\right)^{2} \frac{d^{2} u_{n+1}}{d x^{2}}+2 S_{2}(x) \frac{d u_{n}}{d x} \frac{d^{2} u_{n}}{d x^{2}} \frac{d u_{n+1}}{d x}+f^{\prime}\left(x, u_{n}\right) u_{n+1}= \\
& \frac{d^{2}}{d x^{2}}\left(S_{1}(x) \frac{d^{2} u_{n}}{d x^{2}}\right)+S_{2}(x)\left(\frac{d u_{n}}{d x}\right)^{2} \frac{d^{2} u_{n}}{d x^{2}}+2 S_{2}(x) \frac{d u_{n}}{d x} \frac{d^{2} u_{n}}{d x^{2}} \frac{d u_{n}}{d x}+f^{\prime}\left(x, u_{n}\right) u_{n} .
\end{aligned}
$$


But, using (5)

$$
\frac{d^{2} u_{n}}{d x^{2}}\left(S_{1}(x) \frac{d^{2} u_{n}}{d x^{2}}\right)+S_{2}(x)\left(\frac{d u_{n}}{d x}\right)^{2} \frac{d^{2} u_{n}}{d x^{2}} \approx w(x)-f\left(x, u_{n}\right),
$$

and substituting it into (7), we obtained the differential equation on $u_{n+1}(x)$,

$$
\begin{aligned}
& \frac{d^{2}}{d x^{2}}\left(S_{1}(x) \frac{d^{2} u_{n+1}}{d x^{2}}\right)+S_{2}(x)\left(\frac{d u_{n}}{d x}\right)^{2} \frac{d^{2} u_{n+1}}{d x^{2}}+2 S_{2}(x) \frac{d u_{n}}{d x} \frac{d^{2} u_{n}}{d x^{2}} \frac{d u_{n+1}}{d x}+f^{\prime}\left(x, u_{n}\right) u_{n+1} \\
& =2 S_{2}(x)\left(\frac{d u_{n}}{d x}\right)^{2} \frac{d^{2} u_{n}}{d x^{2}}+f^{\prime}\left(x, u_{n}\right) u_{n}-f\left(x, u_{n}\right)+w(x),
\end{aligned}
$$

which is the sought quasilinear differential equation on $u_{n+1}(x)$.

\section{A numerical discretization method for solving quasilinear differential equations involving up to the 4th order derivative under some conditions}

The developments of this section apply to any quasilinear differential equation involving up to the fourth order derivative, i.e. to any differential equation of the form

$$
m_{4}(x) \frac{d^{4} u}{d x^{4}}+m_{3}(x) \frac{d^{3} u}{d x^{3}}+m_{2}(x) \frac{d^{2} u}{d x^{2}}+m_{1}(x) \frac{d u}{d x}+m_{0}(x) u(x)=n(x)
$$

with a solution $u(x)$ satisfying the boundary conditions (2). Note that by expanding the first term of (8), we get a differential equation of the previous type. However, in our method to solve the BEVKNV differential equation we will not make that expansion in (8). We will use the notation $u^{(l)}(x)=d^{l} u / d x^{l}, 1 \leq l \leq 4$.

In order to solve the differential equation, the idea is to consider an interval $[-R, R]$ for $x$, where $R$ and $N$ are sufficiently large, and partition it into $N$ equally sized subintervals defined by the coordinates $x_{n}=-R+n h, h=2 R / N$, $0 \leq n \leq N$, and, then, to obtain an algebraic equation from the differential equation by discretizing the function $u(x)$ according to the grid $x_{n}, 0 \leq n \leq N$ for $x$ using a vector $\mathbf{u}$ with $\mathbf{u}^{\mathrm{T}}=\left[\begin{array}{llll}u\left(x_{0}\right) & u\left(x_{1}\right) & \cdots & u\left(x_{N}\right)\end{array}\right]$. To obtain the algebraic equation we will obtain approximate algebraic expressions in terms of $\mathbf{u}$ for similar discretizations for

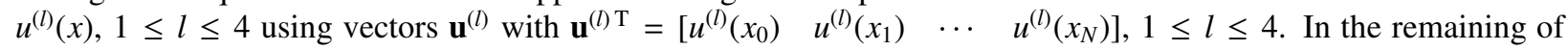
this section, we will obtain those expressions. When dealing with (8), we will make use of the approximate algebraic expressions for the first two derivatives of $u_{n}$ and $u_{n+1}$ in (8) and the first second order derivative in the right-hand side of (8). In our developments we will use Fourier transforms. Given a real valued function $h(x)$, the Fourier transform of $h(x)$ will be denoted as $\mathcal{F}(h(x))$. In a consistent way, given a real valued function $H(\eta)$, the inverse Fourier transform of $H(\eta)$ will be denoted as $\mathcal{F}^{-1}(H(\eta))$. There is more than one definition for the Fourier transform. Here, we will use the definition (see, for instance, [6])

$$
H(\eta)=\mathcal{F}(h(x))=\int_{-\infty}^{\infty} h(x) e^{-i \eta x} d x,
$$

with the corresponding definition for the inverse Fourier transform

$$
\mathcal{F}^{-1}(H(\eta))=\frac{1}{2 \pi} \int_{-\infty}^{\infty} H(\eta) e^{i \eta x} d s
$$

We start by obtaining an approximate algebraic expression for $\mathbf{u}^{(4)}$ in terms of $\mathbf{u}$. Towards that end, consider the trivial equality

$$
u^{(4)}+\alpha u=u^{(4)}+\alpha u,
$$

where $\alpha$ is a constant $>0$. Taking the Fourier transform of both sides and using the differentation rule on the left hand-side, we obtain

$$
\eta^{4} U(\eta)+\alpha U(\eta)=\mathcal{F}\left(u^{(4)}(x)+\alpha u(x)\right),
$$


where $\eta$ is the Fourier transform variable and $U(\eta)$ denotes the Fourier transform of $u(x)$. This gives the expression for $U(\eta)$

$$
U(\eta)=\frac{\mathcal{F}\left(u^{(4)}(x)+\alpha u(x)\right)}{\eta^{4}+\alpha} .
$$

Taking the inverse Fourier transform on both sides, we get

$$
u(x)=\mathcal{F}^{-1}\left(\frac{1}{\eta^{4}+\alpha}\right) *\left(u^{(4)}(x)+\alpha u(x)\right),
$$

where $*$ is convolution product. The inverse Fourier transform of $1 /\left(\eta^{4}+\alpha\right)$ turns out to be

$$
G(x)=\frac{1}{2 \alpha^{3 / 4}} \exp \left(-\frac{\alpha^{1 / 4}}{\sqrt{2}}|x|\right) \sin \left(\frac{\alpha^{1 / 4}}{\sqrt{2}}|x|+\frac{\pi}{4}\right)
$$

and, accordingly, we have

$$
u(x)=\int_{-R}^{R} G(x-t)\left(u^{(4)}(t)+\alpha u(t)\right) d t,
$$

where $R$ is such that the previous integral is sufficiently close to the exact value for $u(x)$, which would be obtained with an integral running from $-\infty$ to $\infty$.

Then, we convert the integral into a summation by using, for instance, the well-known trapezoidal rule with coordinates $x_{n}, 0 \leq n \leq N$, where $N$ is sufficiently large for the error associated with the use of the trapezoidal rule to be sufficiently small. Application of the trapezoidal rule to the evaluation of $u\left(x_{n}\right), 0 \leq n \leq N$ using (9) yields

$$
u\left(x_{n}\right)=\sum_{m=0}^{N} G\left(x_{n}-x_{m}\right)\left(u^{(4)}\left(x_{m}\right)+\alpha u\left(x_{m}\right)\right) b_{m}, \quad 0 \leq n \leq N,
$$

where $b_{0}=h / 2, b_{m}=h, 1 \leq m \leq N-1$, and $b_{N}=h / 2$. Introducing the vector $\mathbf{b}=\left(b_{m}\right)_{0 \leq m \leq N}$, the matrix

$$
\mathbf{G}=\left[\begin{array}{cccc}
G(0) & G(-h) & \cdots & G(-N h) \\
G(h) & G(0) & \cdots & G(-(N-1) h) \\
\vdots & \vdots & \ddots & \ldots \\
G(N h) & G((N-1) h) & \cdots & G(0)
\end{array}\right],
$$

and with $\operatorname{diag}[\mathbf{x}]$ denoting the matrix with main diagonal including the elements of vector $\mathbf{x}$ and 0 's elsewhere, we get the vector expression

$$
\mathbf{u}=\mathbf{G} \operatorname{diag}[\mathbf{b}]\left(\mathbf{u}^{(4)}+\alpha \mathbf{u}\right)
$$

and, by isolating $\mathbf{u}^{(4)}$,

$$
\mathbf{u}^{(4)}=\left(\operatorname{diag}[\mathbf{b}]^{-1} \mathbf{G}^{-1}-\alpha \mathbf{I}\right) \mathbf{u},
$$

where $\mathbf{I}$ is an identity matrix of appropriate dimension and where the existence of the inverse of $\mathbf{G}$ is proved in the Appendix for any discretized Green function $\mathbf{G}$ on a finite interval.

In order to obtain approximate expressions for $\mathbf{u}^{(l)}, 1 \leq l \leq 3$ in terms of $\mathbf{u}$, we consider approximations for the three first order derivatives of $u$ by taking derivatives with respect to $x$ in (9):

$$
u^{(l)}(x)=\int_{-R}^{R} \frac{d^{l} G(x-t)}{d x^{l}}\left(u^{(4)}(t)+\alpha u(t)\right) d t .
$$


Here, again, $R$ has to be large enough for the error in the integral with respect to the exact value which would be obtained with an integral running from $-\infty$ to $\infty$ to be sufficiently small. The following expression can be found for $d^{l} G(x) / d x^{l}, 1 \leq l \leq 3$

$$
\frac{d^{l} G(x)}{d x^{l}}=\frac{(-\operatorname{sign}(x))^{l}}{2 \alpha^{3-l}} e^{-\frac{\alpha}{\sqrt{2}}|x|} \sin \left(\frac{\alpha}{\sqrt{2}}|x|-(l-1) \frac{\pi}{4}\right),
$$

where $\operatorname{sign}(x)=1$ for $x \geq 0$ and 0 otherwise. Replacing $x$ by $x-t$ gives the following expression for $d^{l} G(x-t) / d x^{l}$, $1 \leq l \leq 3$, to be used in conjunction with (11)

$$
\frac{d^{l} G(x-t)}{d x^{l}}=\frac{(-\operatorname{sign}(x-t))^{l}}{2 \alpha^{3-l}} e^{-\frac{\alpha}{\sqrt{2}}|x|} \sin \left(\frac{\alpha}{\sqrt{2}}|x-t|-(l-1) \frac{\pi}{4}\right) .
$$

Then, we discretize (11) as done to obtain (10), with the result

$$
\mathbf{u}^{(l)}=\mathbf{G}^{(l)} \operatorname{diag}[\mathbf{b}]\left(\mathbf{u}^{(4)}+\alpha \mathbf{u}\right),
$$

where

$$
\mathbf{G}^{(l)}=\left[\begin{array}{cccc}
\frac{d^{l} G}{d x^{l}}(0) & \frac{d^{l} G}{d x^{l}}(-h) & \ldots & \frac{d^{l} G}{d x^{l}}(-N h) \\
\frac{d^{l} G}{d x^{l}}(h) & \frac{d^{l} G}{d x^{l}}(0) & \ldots & \frac{d^{l} G}{d x^{l}} G(-(N-1) h) \\
\vdots & \vdots & \ddots & \ldots \\
\frac{d^{l} G}{d x^{l}}(N h) & \frac{d^{l} G}{d x^{l}}((N-1) h) & \ldots & \frac{d^{l} G}{d x^{l}}(0)
\end{array}\right] .
$$

Introducing matrix $\mathbf{A}^{(l)}=\mathbf{G}^{(l)} \operatorname{diag}[\mathbf{b}], 1 \leq l \leq 3$, we obtain

$$
\mathbf{u}^{(l)}=\mathbf{A}^{(l)}\left(\mathbf{u}^{(4)}+\alpha \mathbf{u}\right) \text {. }
$$

Introducing matrix $\mathbf{A}=\mathbf{G} \operatorname{diag}[\mathbf{b}]$, we can rewrite (10) as

$$
\mathbf{u}^{(4)}=\left(\mathbf{A}^{-1}-\alpha \mathbf{I}\right) \mathbf{u} .
$$

By substituting the second equation into the first, we get, for $1 \leq l \leq 3$,

$$
\mathbf{u}^{(l)}=\mathbf{A}^{(l)}\left(\alpha \mathbf{u}+\left(\mathbf{A}^{-1}-\alpha \mathbf{I}\right) \mathbf{u}\right)=\mathbf{A}^{(l)} \mathbf{A}^{-1} \mathbf{u} .
$$

Finally, using $\mathbf{A}^{(l)}=\mathbf{G}^{(l)} \operatorname{diag}[\mathbf{b}]$ and $\mathbf{A}=\mathbf{G} \operatorname{diag}[\mathbf{b}]$, we obtain, for $1 \leq l \leq 3$,

$$
\begin{aligned}
\mathbf{u}^{(l)} & =\mathbf{G}^{(l)} \operatorname{diag}[\mathbf{b}](\mathbf{G} \operatorname{diag}[\mathbf{b}])^{-1} \mathbf{u} \\
& =\mathbf{G}^{(l)} \operatorname{diag}[\mathbf{b}] \operatorname{diag}[\mathbf{b}]^{-1} \mathbf{G}^{-1} \mathbf{u}=\mathbf{G}^{(l)} \mathbf{G}^{-1} \mathbf{u},
\end{aligned}
$$

which is the sought approximate expression for $\mathbf{u}^{(l)}, 1 \leq l \leq 3$ in terms of $\mathbf{u}$.

We can summarize the results obtained in this section regarding the approximate alebraic expressions for $\mathbf{u}^{(l)}$, $1 \leq l \leq 4$ in terms of $\mathbf{u}$ as

$$
\mathbf{u}^{(l)}=\mathbf{D}_{l} \mathbf{u},
$$

where

$$
\mathbf{D}_{l}=\mathbf{G}^{(l)} \mathbf{G}^{-1}, \quad 1 \leq l \leq 3
$$

and (10)

$$
\mathbf{D}_{4}=\operatorname{diag}[\mathbf{b}]^{-1} \mathbf{G}^{-1}-\alpha \mathbf{I} .
$$




\section{Method for solving the BEVKNV differential equation}

The method for solving the BEVKNV differential Eq. (1) combines QLM with the discretization of the quasilinear differential equation on $u_{n+1}(x)$ (8). Using the discretization method obtained in Section 2 for solving quasilinear differential equation applied to $(8)$, we obtain

$$
\begin{aligned}
& \mathbf{D}_{2} \operatorname{diag}\left[\mathbf{S}_{1}\right] \mathbf{D}_{2} \mathbf{u}_{n+1}+\operatorname{diag}\left[\mathbf{S}_{2} \odot \mathbf{D}_{1} \mathbf{u}_{n} \odot \mathbf{D}_{2} \mathbf{u}_{n}\right] \mathbf{D}_{2} \mathbf{u}_{n+1}+2 \operatorname{diag}\left[\mathbf{S}_{2} \odot\left(\mathbf{D}_{1} \mathbf{u}_{n}\right) \odot\left(\mathbf{D}_{2} \mathbf{u}_{n}\right)\right] \mathbf{D}_{1} \mathbf{u}_{n+1} \\
& +\operatorname{diag}\left[\mathbf{f}^{\prime}\left(\mathbf{x}, \mathbf{u}_{n}\right)\right] \mathbf{u}_{n+1}=2 \mathbf{S}_{2} \odot \mathbf{D}_{1} \mathbf{u}_{n} \odot \mathbf{D}_{1} \mathbf{u}_{n} \odot \mathbf{D}_{2} \mathbf{u}_{n}+\mathbf{f}^{\prime}\left(\mathbf{x}, \mathbf{u}_{n}\right) \odot \mathbf{u}_{n}-\mathbf{f}\left(\mathbf{x}, \mathbf{u}_{n}\right)+\mathbf{w},
\end{aligned}
$$

where $\odot$ represents element-wise multiplication between two vectors of same length, where

$$
\begin{aligned}
& \mathbf{u}_{n}=\left(u_{n}\left(x_{m}\right)\right)_{0 \leq m \leq N}, \\
& \mathbf{u}_{n+1}=\left(u_{n+1}\left(x_{m}\right)\right)_{0 \leq m \leq N}, \\
& \mathbf{D}_{1}=\mathbf{G}^{(1)} \mathbf{G}^{-1}, \\
& \mathbf{D}_{2}=\mathbf{G}^{(2)} \mathbf{G}^{-1}, \\
& \mathbf{S}_{1}=\left(S_{1}\left(x_{m}\right)\right)_{0 \leq m \leq N}, \\
& \mathbf{S}_{2}=\left(S_{2}\left(x_{m}\right)\right)_{0 \leq m \leq N}, \\
& \mathbf{w}=\left(w\left(x_{m}\right)\right)_{0 \leq m \leq N}, \\
& \mathbf{f}\left(\mathbf{x}, \mathbf{u}_{n}\right)=\left(f\left(x_{m}, u_{n}\left(x_{m}\right)\right)\right)_{0 \leq m \leq N}, \\
& \mathbf{f}^{\prime}\left(\mathbf{x}, \mathbf{u}_{n}\right)=\left(f^{\prime}\left(x_{m}, u_{n}\left(x_{m}\right)\right)\right)_{0 \leq m \leq N},
\end{aligned}
$$

where $x_{n}=-R+n h, h=2 R / N, 0 \leq n \leq N$, and where $R$ and $N$ are sufficiently large for the discretization error to be sufficiently small. The implementation of our proposed iterative method is given in Algorithm 1

\section{Accuracy of discretization of the derivatives}

To test the accuracy of the discretizations of derivatives defined in Section 3 , we selected the function $u(x)=e^{-x^{2}}$, which can be a solution of (1).

All the results given in this section were obtained taking $R=5$. Fig. 1 gives the plots of that function and its first four derivatives. We took $N=499$ and computed the difference between the computed four first order derivatives at the grid $x_{n}, 0 \leq n \leq N$ and the exact values of those derivatives. The results are given in Fig. 2. We can note that the accuracy in the derivatives is good except for the third order derivative. This is an essential limitation of the method we could overcome in future work. Table 1 gives, for $1 \leq l \leq 4,\left\|\mathbf{D}_{l} \mathbf{u}-\mathbf{u}^{(l)}\right\|_{\infty}$, which is the maximum absolute error in the $l$ th order derivative at the grid points, for $N=499$ and varying $\alpha$. We will comment on the impact of $\alpha$ in the accuracy of the first and second order derivatives. The reason is that when applying the discretization method to the solution of the BEVKNV differential equation only the discretization of those derivatives will be used. We can see that the best results are obtained for $\alpha \leq 1$ and that under that restriction the accuracy depends very little on the particular value of $\alpha$. In order to analyze the impact of the number of grid points $N$ on the accuracy in the first and second order derivatives with a good value for $\alpha$, Table 2 gives, for $1 \leq l \leq 4,\left\|\mathbf{D}_{l} \mathbf{u}-\mathbf{u}^{(l)}\right\|_{\infty}$ as a function of $N$ for $\alpha=0.1$. The results show that as the number of grid points is increased the accuracy improves but there is a point beyond which there is no significant change on the accuracy. A reasonable selection for $N$ for $u(x)=e^{-x^{2}}$ would be $N=499$.

\section{Numerical experimentation of the method for solving the BEVKNV differential equation}

In this section, we will analyze the convergence and accuracy of the method proposed in this paper for the solution of the BEVKNV differential equation in nine cases. We designed nine different cases. The purpose of the first four cases is to check the validity and accuracy of our proposed iterative method. The remaining five cases give us vertical deflection of beam when rectangular load is applied under the influence of different combinations of parameters. In the first 4 cases, $u(x), E(x) I(x), E(x) A(x)$, and $f(x, u)$ will be known and the applied load will be chosen so that the 


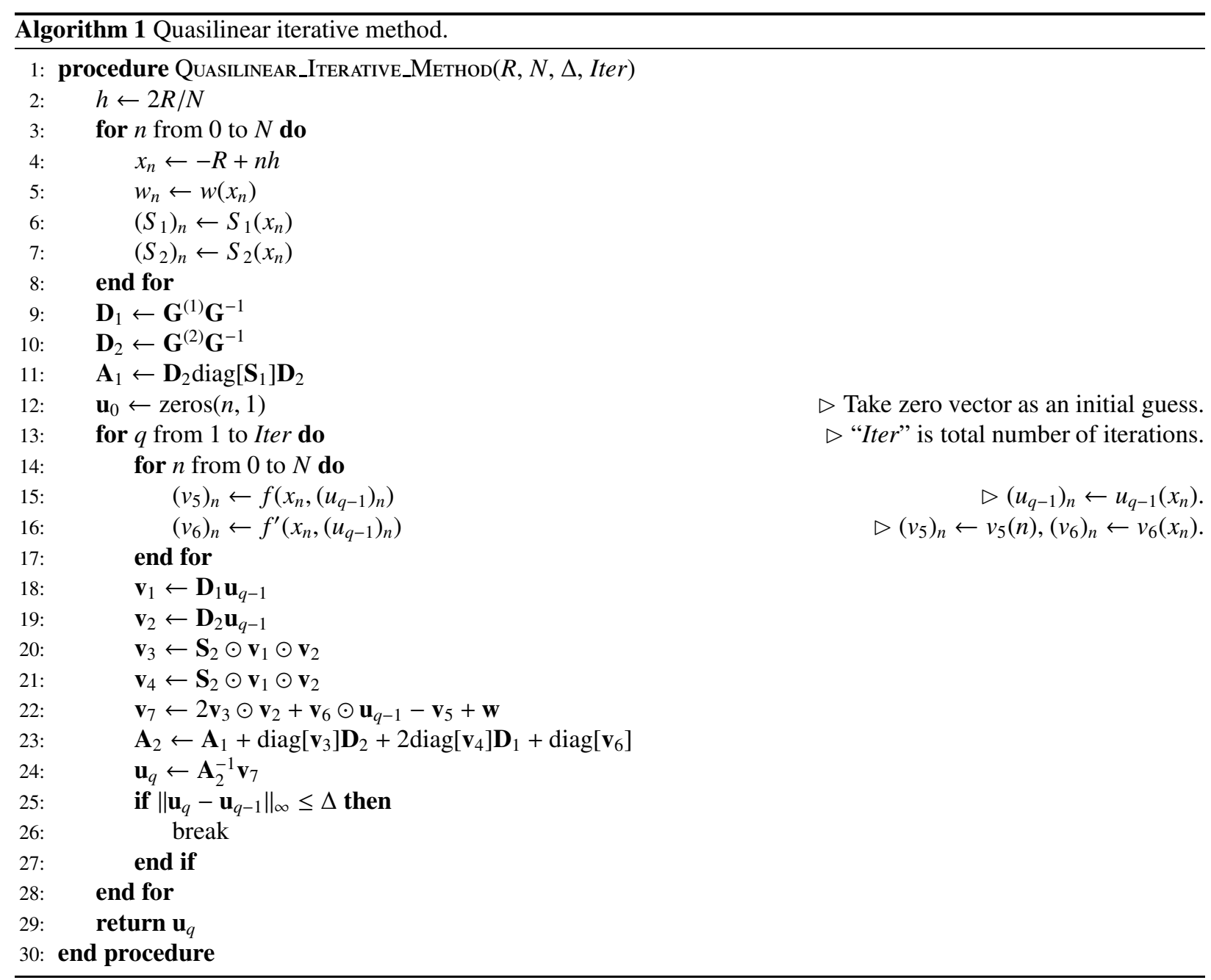

Table 1: Maximum absolute error in the derivatives for $N=499$ and varying $\alpha$.

\begin{tabular}{|c|c|c|c|c|c|c|c|}
\hline$\alpha$ & 1,000 & 100 & 10 & 1 & $10^{-1}$ & $10^{-2}$ & $10^{-3}$ \\
\hline$\left\|\mathbf{D}_{1} \mathbf{u}-\mathbf{u}^{(1)}\right\|_{\infty}$ & $8 \times 10^{-7}$ & $10^{-7}$ & $4 \times 10^{-8}$ & $3 \times 10^{-8}$ & $3 \times 10^{-8}$ & $3 \times 10^{-8}$ & $3 \times 10^{-8}$ \\
$\left\|\mathbf{D}_{2} \mathbf{u}-\mathbf{u}^{(2)}\right\|_{\infty}$ & $3 \times 10^{-2}$ & $4 \times 10^{-3}$ & $7 \times 10^{-4}$ & $4 \times 10^{-4}$ & $4 \times 10^{-4}$ & $4 \times 10^{-4}$ & $4 \times 10^{-4}$ \\
$\left\|\mathbf{D}_{3} \mathbf{u}-\mathbf{u}^{(3)}\right\|_{\infty}$ & 10 & 1 & 0.2 & 0.1 & 0.1 & 0.1 & 0.1 \\
$\left\|\mathbf{D}_{4} \mathbf{u}-\mathbf{u}^{(4)}\right\|_{\infty}$ & $2 \times 10^{-4}$ & $7 \times 10^{-6}$ & $8 \times 10^{-6}$ & $9 \times 10^{-6}$ & $9 \times 10^{-6}$ & $9 \times 10^{-6}$ & $10^{-5}$ \\
\hline
\end{tabular}

Table 2: Maximum absolute error in the derivatives for $\alpha=0.1$ and varying $N$.

\begin{tabular}{|c|c|c|c|c|c|c|c|}
\hline$N+1$ & 100 & 200 & 300 & 400 & 500 & 600 & 800 \\
\hline$\left\|\mathbf{D}_{1} \mathbf{u}-\mathbf{u}^{(1)}\right\|_{\infty}$ & $2 \times 10^{-5}$ & $10^{-6}$ & $2 \times 10^{-7}$ & $7 \times 10^{-8}$ & $3 \times 10^{-8}$ & $10^{-8}$ & $3 \times 10^{-8}$ \\
$\left\|\mathbf{D}_{2} \mathbf{u}-\mathbf{u}^{(2)}\right\|_{\infty}$ & 0.01 & 0.003 & 0.001 & $6 \times 10^{-4}$ & $4 \times 10^{-4}$ & $3 \times 10^{-4}$ & $2 \times 10^{-4}$ \\
$\left\|\mathbf{D}_{3} \mathbf{u}-\mathbf{u}^{(3)}\right\|_{\infty}$ & 0.6 & 0.3 & 0.2 & 0.21 & 0.1 & 0.1 & 0.08 \\
$\left\|\mathbf{D}_{4} \mathbf{u}-\mathbf{u}^{(4)}\right\|_{\infty}$ & $2 \times 10^{-4}$ & $10^{-5}$ & $4 \times 10^{-6}$ & $6 \times 10^{-6}$ & $9 \times 10^{-6}$ & $10^{-5}$ & $2 \times 10^{-5}$ \\
\hline
\end{tabular}




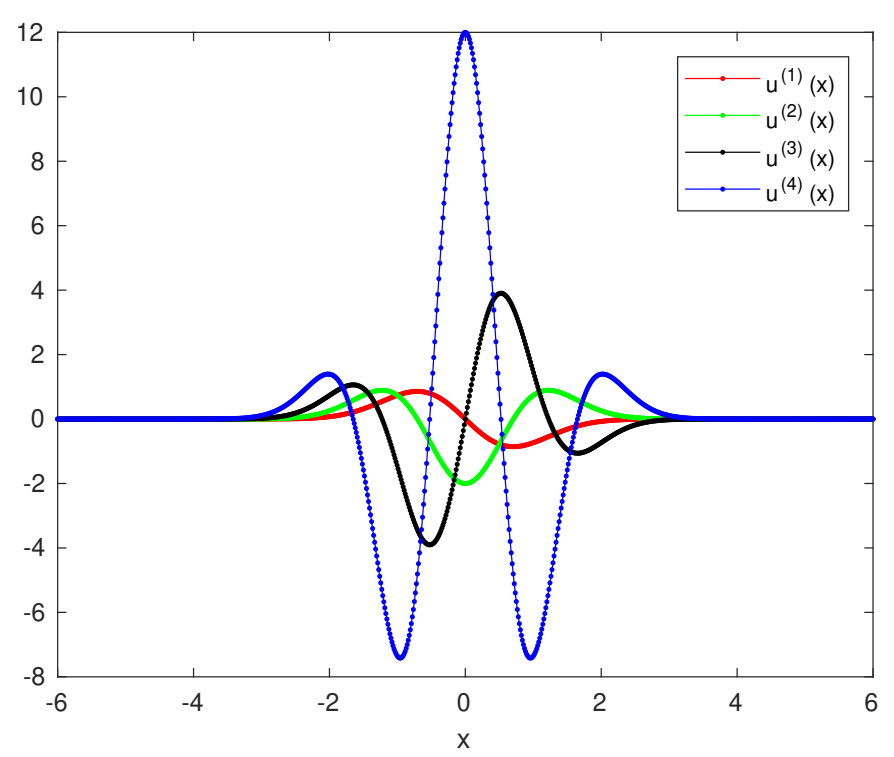

Figure 1: Plot of $e^{-x^{2}}$ and its four first derivatives.

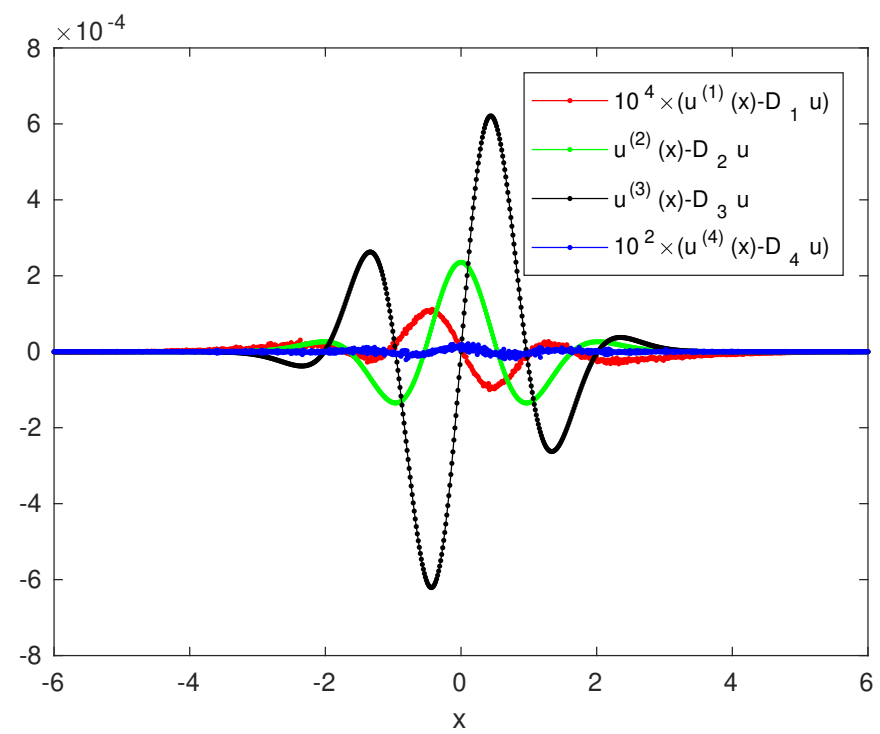

Figure 2: Errors in the four first derivatives of $e^{-x^{2}}$. 
Table 3: First four cases considered for the numerical experimentation of the method.

\begin{tabular}{|r|cccccccc|}
\hline case & $u(x)$ & $\lambda_{0}$ & $\lambda_{1}$ & $\lambda_{2}$ & $\lambda_{3}$ & $\lambda_{4}$ & $\gamma_{1}$ & $\gamma_{2}$ \\
\hline 1 & $e^{-x^{2}}$ & 12 & $18 / 5$ & 1 & $6 / 25$ & 1 & $2+\sin (x)$ & $5+\cos (x)$ \\
2 & $e^{-x^{2}}$ & 1 & 0.05 & 0.1 & 0 & 3 & 6 & 1 \\
3 & $\cos (x) e^{-x^{2}}$ & 1 & 0.05 & 0.1 & 0 & 3 & 6 & 1 \\
4 & $\sin (x) e^{-x^{2}}$ & 1 & 0.05 & 0.1 & 0 & 3 & 6 & 1 \\
\hline
\end{tabular}

Table 4: Last five cases considered for the numerical experimentation of the method.

\begin{tabular}{|r|cccccccc|}
\hline case & $w(x)$ & $\lambda_{0}$ & $\lambda_{1}$ & $\lambda_{2}$ & $\lambda_{3}$ & $\lambda_{4}$ & $\gamma_{1}$ & $\gamma_{2}$ \\
\hline 5 & $3 \times \mathbf{1}_{x \leq 3}$ & 1 & 0.05 & 0.1 & 0 & 3 & 6 & 1 \\
6 & $6 \times \mathbf{1}_{x \leq 3}$ & 1 & 0.05 & 0.1 & 0 & 3 & 6 & 1 \\
7 & $9 \times \mathbf{1}_{x \leq 3}$ & 1 & 0.05 & 0.1 & 0 & 3 & 6 & 1 \\
8 & $6 \times \mathbf{1}_{x \leq 3}$ & 1 & 0.05 & 0.1 & 0 & 3 & - & - \\
9 & $6 \times \mathbf{1}_{x \leq 3}$ & 1 & - & - & 0 & 3 & 6 & 1 \\
\hline
\end{tabular}

selected $u(x)$ is a solution of the BEVKNV differential equation. In the remaining cases, $E(x) I(x), E(x) A(x), f(x, u)$, and $w(x)$ will be known and $u(x)$ will be unknown. The function $E(x) I(x)$ will have in all cases the form

$$
E(x) I(x)=\frac{1}{12}\left(\lambda_{0}+\lambda_{1} e^{-\lambda_{2} x^{2}}+\lambda_{3} e^{-2 x^{2}}\right)^{\lambda_{4}} .
$$

The function $E(x) A(x)$ will have in all cases the form

$$
E(x) A(x)=1+\lambda_{1} e^{-\lambda_{2} x^{2}} .
$$

Finally, the function $f(x, u)$ will have in all cases the form

$$
f(x, u)=\gamma_{1} u(x)+\gamma_{2} u(x)^{3} .
$$

Table 3 gives the functions $u(x)$ and the values of the parameters $\lambda_{i}, 0 \leq i \leq 4, \gamma_{1}$ and $\gamma_{2}$ corresponding to the first four cases.

Table 4 gives the functions $w(x)$ and the values of the parameters $\lambda_{i}, 0 \leq i \leq 4, \gamma_{1}$ and $\gamma_{2}$ corresponding to the last five cases. The values of $\gamma_{1}$ and $\gamma_{2}$ for case 8 are left unspecified because we will vary the values of those parameters in that case. Similarly, the values of $\lambda_{1}$ and $\lambda_{2}$ for case 9 are left unspecified because we will also vary the values of those parameters in that case. In the table, $\mathbf{1}_{c}$ denotes the indicator function returning value 1 if condition $c$ is true and value 0 otherwise.

Because of the discretization of the quasilinear differential equation, there is an optimal accuracy which cannot be improved with further iterations of QLM. In all cases, we took a value for $N$ large enough for that accuracy of the method to settle in the range of its maximum value. That optimal accuracy is not only determined by round-off errors but also by the limited accuracy obtained in the derivatives by discretization. The value we took for $N$ is $N=499$ in all cases. Regarding the parameter $\alpha$, according to the numerical experiments reported in the previous section, we took $\alpha=0.1$ in all cases.

Fig. 3 gives the numerical solution for case 1. We run our method with $R=5$ and a tolerance $\Delta=7 \times 10^{-5}$. The method converged to that tolerance in 6 iterations. Fig. 4 plots the absolute errors (difference between the computed value and the exact value) for the discretized computed solution. We can note that absolute errors are always below the specified tolerance. Fig. 5 gives the numerical solution for case 2. We run our method with $R=5$ and a tolerance $\Delta=3.5 \times 10^{-5}$. The method converged to that tolerance in 4 iterations. Fig. 5 plots the absolute errors for the discretized computed solution (Fig. 6). We can note that, as for case 1, the absolute errors are always below the 
specified tolerance. Fig. 7 gives the numerical solution for case 3 . We run our method with $R=5$ and a tolerance $\Delta=8 \times 10^{-5}$. The method converged to that tolerance in 4 iterations. Fig. 5 plots the absolute errors for the discretized computed solution (Fig. 8). The absolute errors are again below the specified tolerance. Fig. 9 gives the numerical solution for case 4. We run our method with $R=5$ and a tolerance $\Delta=1.2 \times 10^{-5}$. The method converged to that tolerance in 3 iterations. Fig. 9 plots the absolute errors for the discretized computed solution. The absolute errors are below the specified tolerance (Fig. 10).

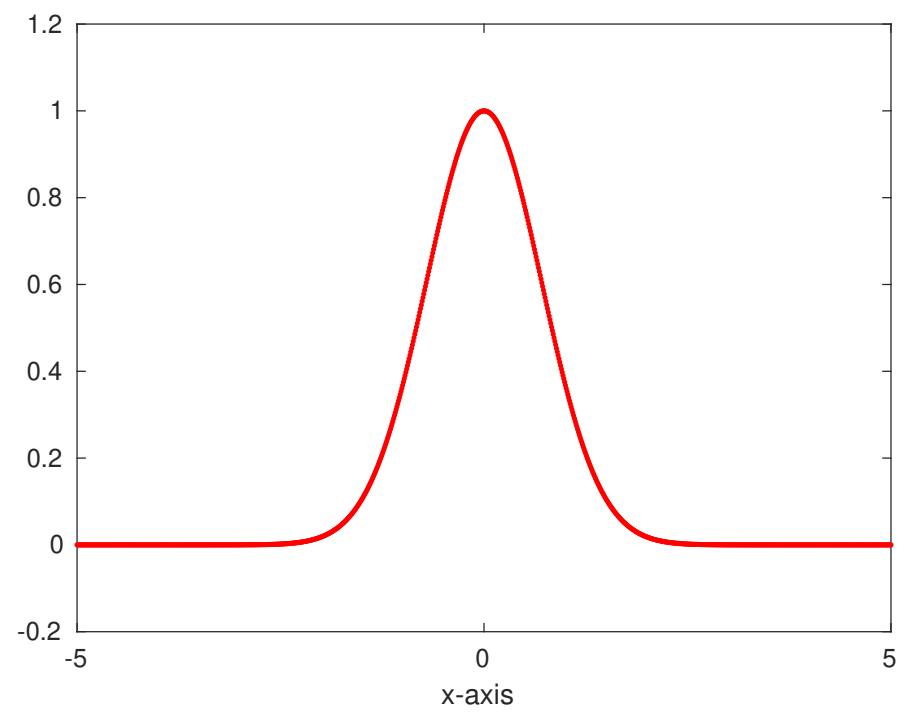

Figure 3: Exact solution for case 1.

For case 5, we run our method with $\alpha=0.1, R=5, N=499$, and tolerances chosen so that the number of iterations of the method were 1, 2, 3, 4, 5, and 6. Fig. 11 plots the computed solution after 6 iterations. Fig. 12 gives $\log _{10}\left(\left\|\mathbf{u}_{n+1}-\mathbf{u}_{n}\right\|_{\infty}\right)$ for $n=0,1,2,3,4,5$. We can note that as $n$ increases $\left\|\mathbf{u}_{n+1}-\mathbf{u}_{n}\right\|_{\infty}$ is reduced significantly till reaching a value $1.56 \times 10^{-10}$ for $n=5$, which is very small compared to the maximum absolute value of $u(x)$, which is $\approx 0.5$.

For case 6 , we also run our method with $\alpha=0.1, R=5, N=499$, and tolerances chosen so that the number of iterations of the method were 1, 2, 3, 4, 5, and 6. Fig. 13 plots the computed solution after 6 iterations. Fig. 14 gives $\log _{10}\left(\left\|\mathbf{u}_{n+1}-\mathbf{u}_{n}\right\|_{\infty}\right)$ for $n=0,1,2,3,4,5$. We can note that, also in this case, as $n$ increases $\left\|\mathbf{u}_{n+1}-\mathbf{u}_{n}\right\|_{\infty}$ is reduced significantly. In this case, the final value for $\left\|\mathbf{u}_{n+1}-\mathbf{u}_{n}\right\|_{\infty}$ is $2.62 \times 10^{-10}$, which is, again, very small compared to the maximum absolute value of $u(x)$, which is $\approx 0.9$.

Case 7 is identical to cases 5 and 6 except for the height of the load function $w(x)$, and we also run our method with $\alpha=0.1, R=5, N=499$, and tolerances chosen so that the number of iterations of the method ranged from 1 to 10. Fig. 15 plots the computed solution after 10 iterations. Fig. 16 gives $\log _{10}\left(\left\|\mathbf{u}_{n+1}-\mathbf{u}_{n}\right\|_{\infty}\right)$ for $n$ ranging from 0 to 9. We can note that, also in this case, as $n$ increases $\left\|\mathbf{u}_{n+1}-\mathbf{u}_{n}\right\|_{\infty}$ is reduced significantly. In this case, the final value for $\left\|\mathbf{u}_{n+1}-\mathbf{u}_{n}\right\|_{\infty}$ is $4.85 \times 10^{-10}$, which is, again, very small compared to the maximum absolute value of $u(x)$, which is $\approx 1.2$.

In case $8, \gamma_{1}$ and $\gamma_{2}$ take four different combinations of values. These correspond to $\gamma_{1}=1$ and $\gamma_{2} / \gamma_{1}=0,1 / 8,1 / 4$, and $1 / 2$. We run our method for each combination with $\alpha=0.1, R=10, N=499$, and tolerances chosen so that the number of iterations of the method ranges from 1 to 10. Fig. 17 plots the computed solutions after 10 iterations. Fig. 18 gives $\log _{10}\left(\left\|\mathbf{u}_{n+1}-\mathbf{u}_{n}\right\|_{\infty}\right)$ for $n$ ranging from 0 to 9 . We can note that as $n$ increases $\left\|\mathbf{u}_{n+1}-\mathbf{u}_{n}\right\|_{\infty}$ is reduced significantly till reaching a value of the order of $10^{-10}$ for $n=9$, which is very small compared to the maximum absolute value of $u(x)$, which is between 2 and 9.

In case $9, \lambda_{1}$ and $\lambda_{2}$ take three different combinations of values. These correspond to $\lambda_{1}=0.05$ and $\lambda_{2} / \lambda_{1}=1,3$ and 10. We run our method for each combination with $\alpha=0.1, R=10, N=499$, and tolerances chosen so that the 


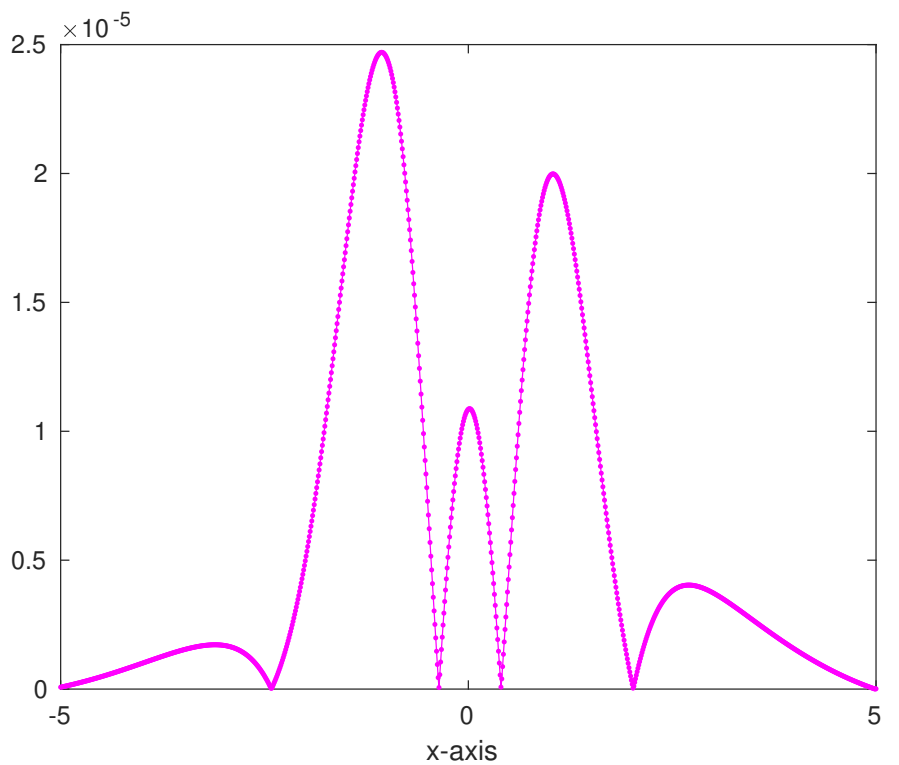

Figure 4: Absolute errors for case 1.

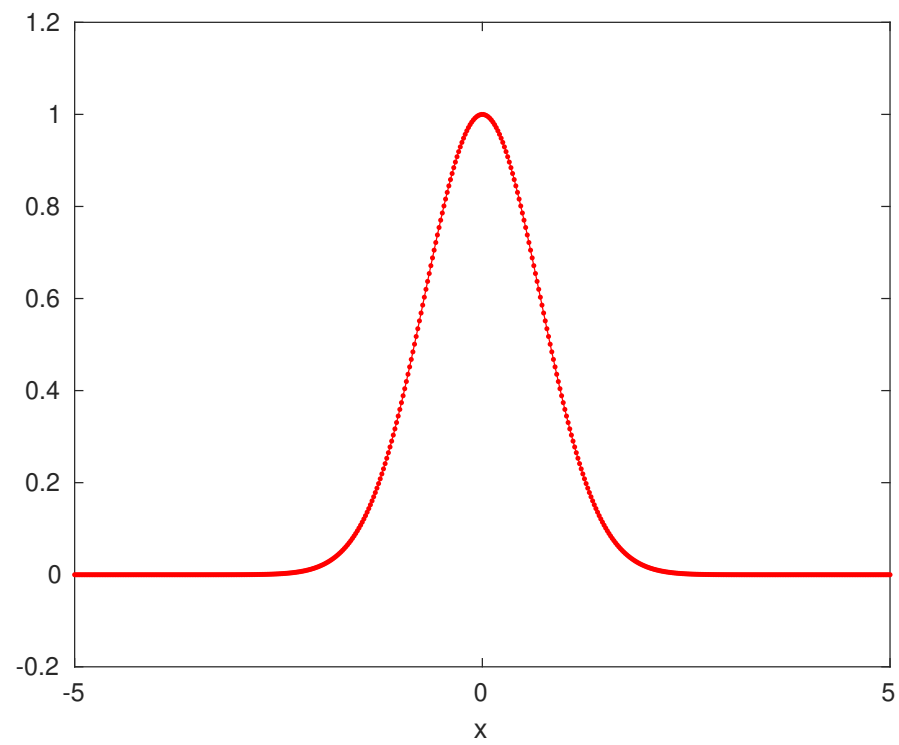

Figure 5: Exact solution for case 2. 


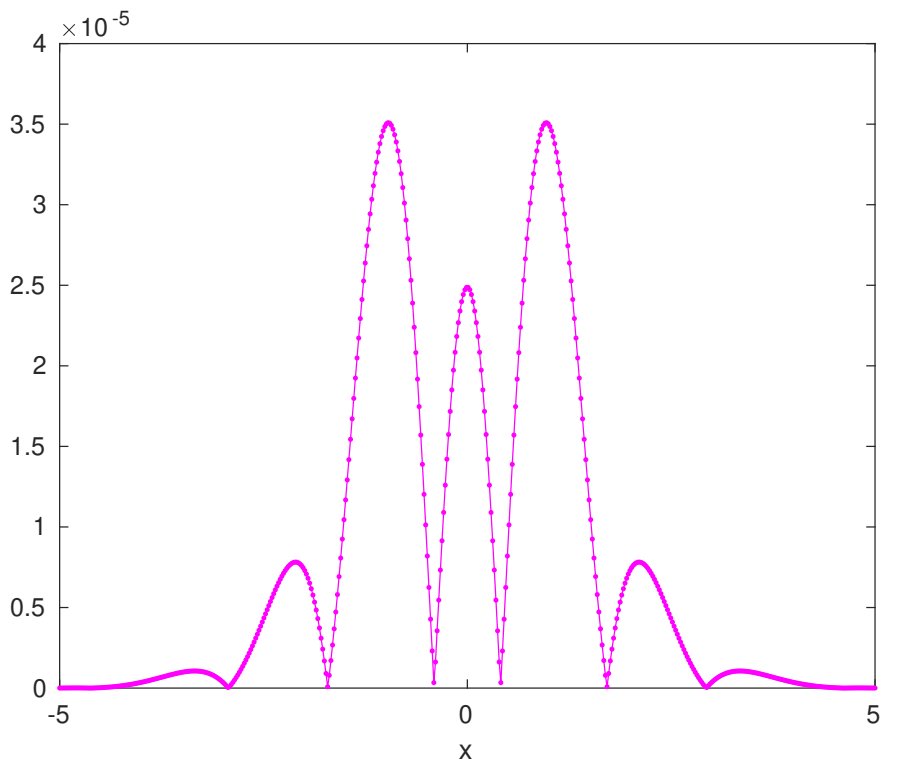

Figure 6: Absolute errors for case 2.

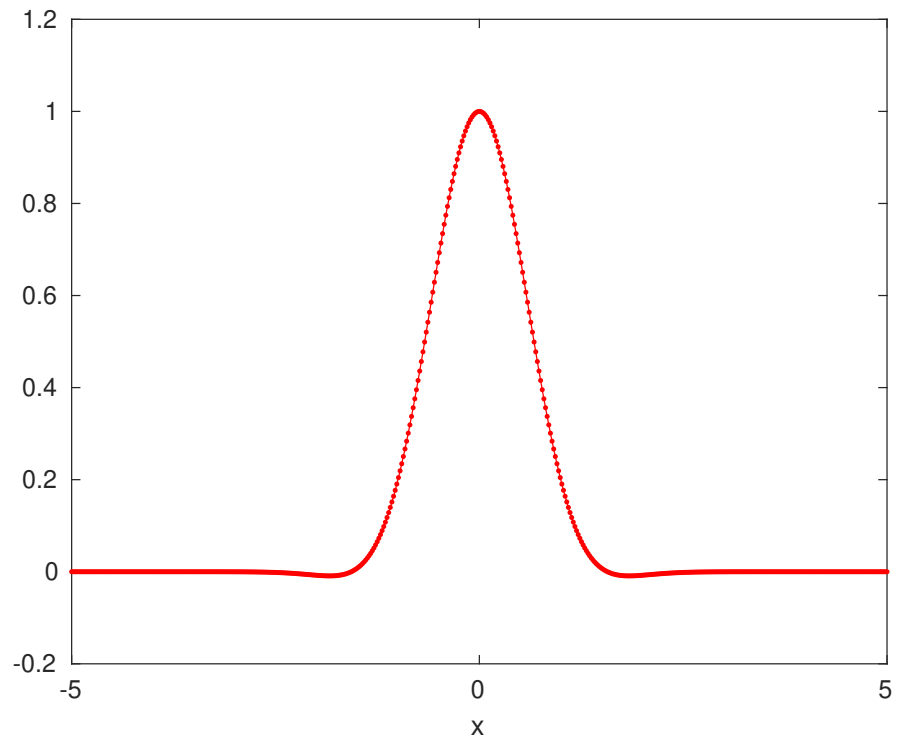

Figure 7: Exact solution for case 3. 


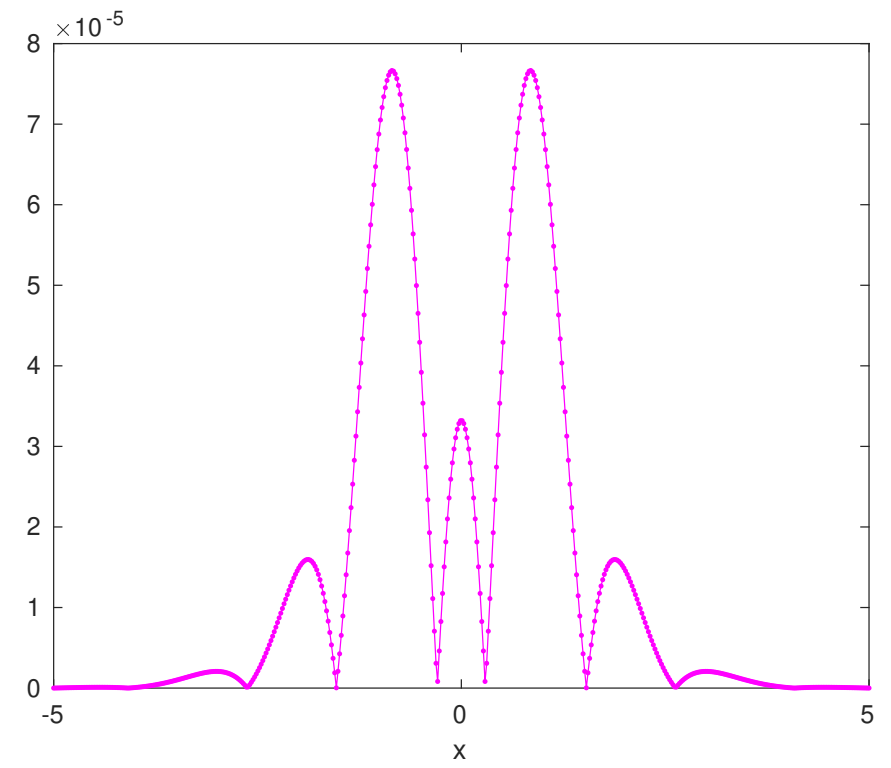

Figure 8: Absolute errors for case 3.

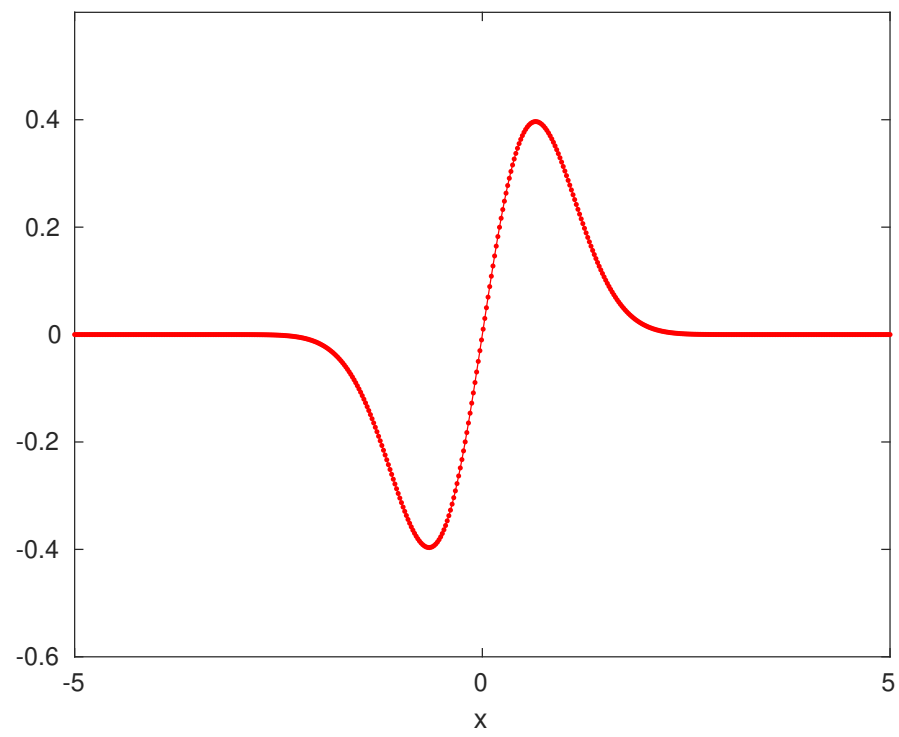

Figure 9: Exact solution for case 4. 


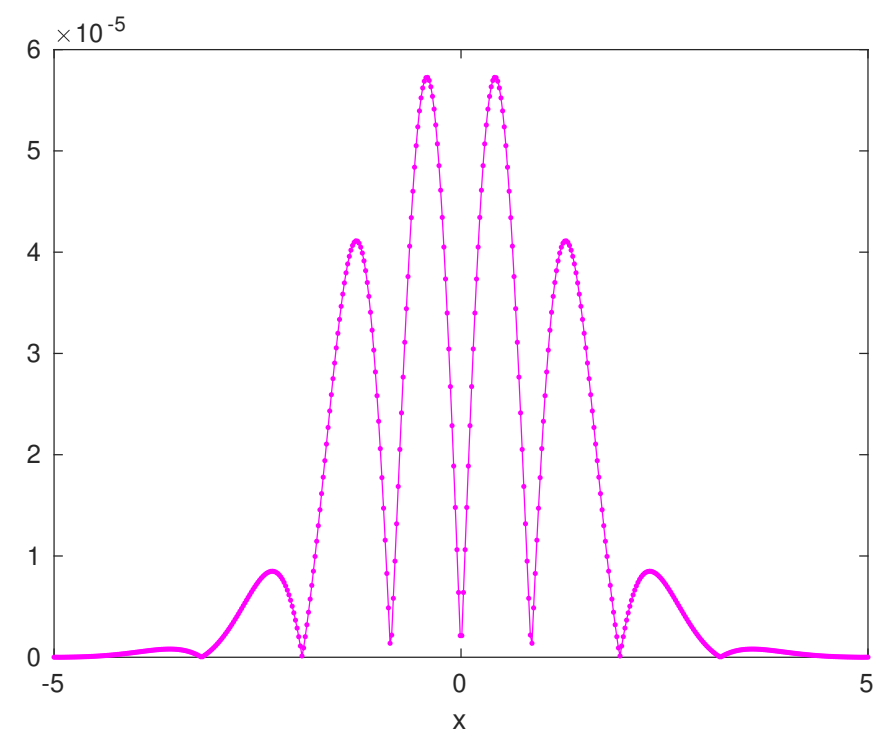

Figure 10: Absolute errors for case 4.

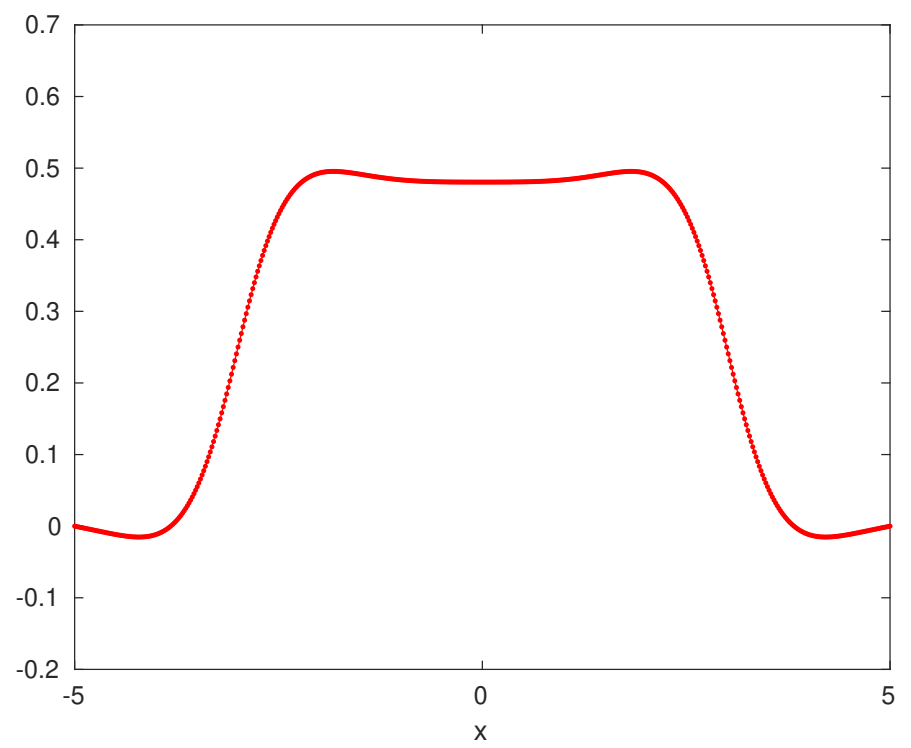

Figure 11: Solution for case 5 after 6 iterations. 


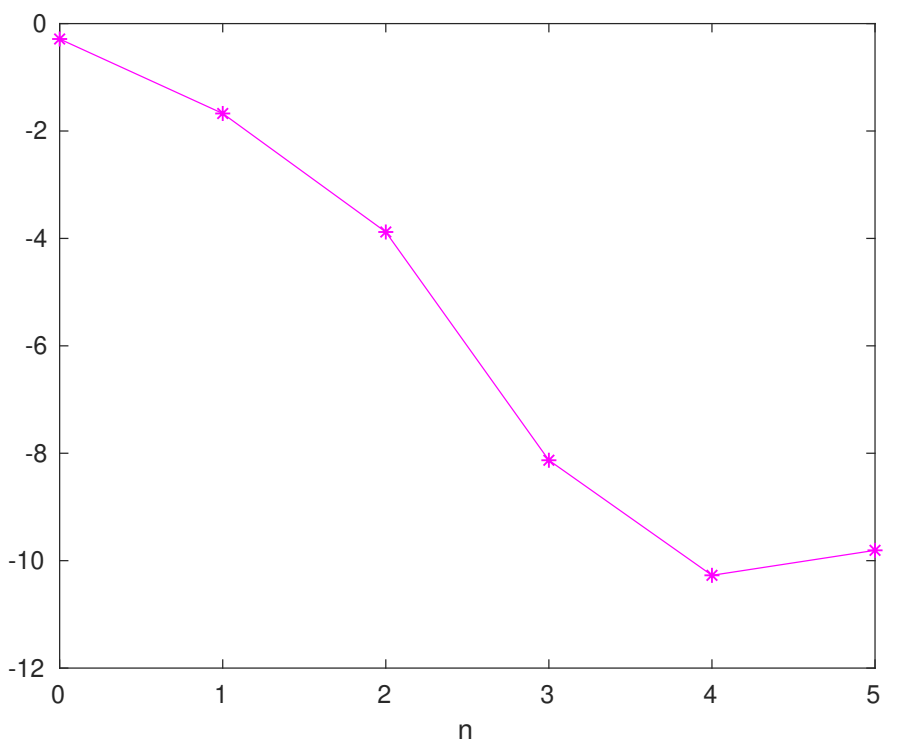

Figure 12: $\log _{10}\left(\left\|\mathbf{u}_{n+1}-\mathbf{u}_{n}\right\|_{\infty}\right)$ for case 5 .

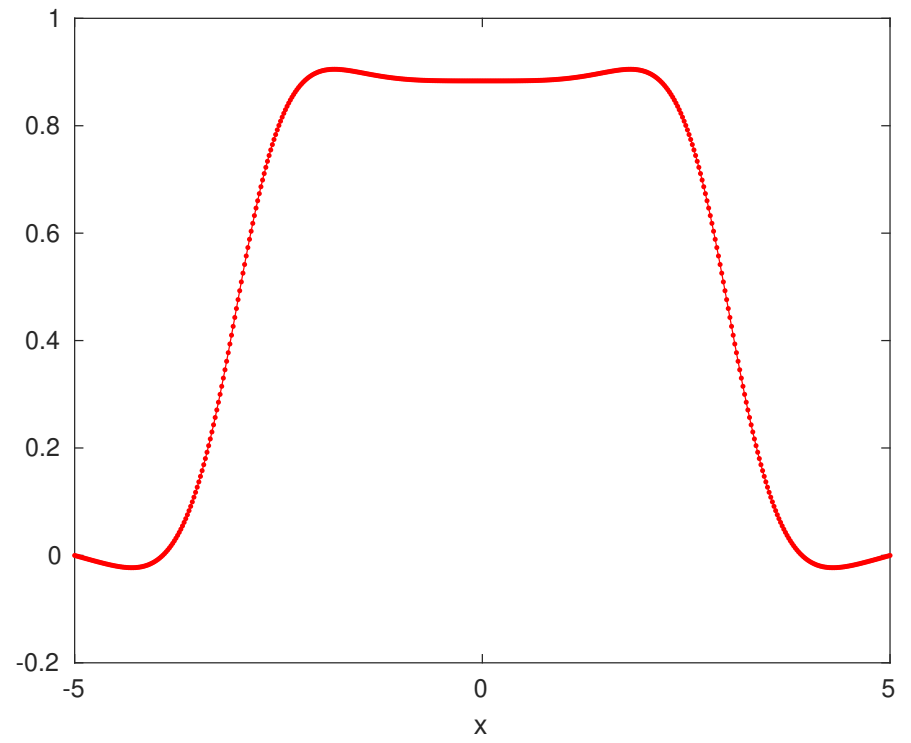

Figure 13: Solution for case 6 after 6 iterations 


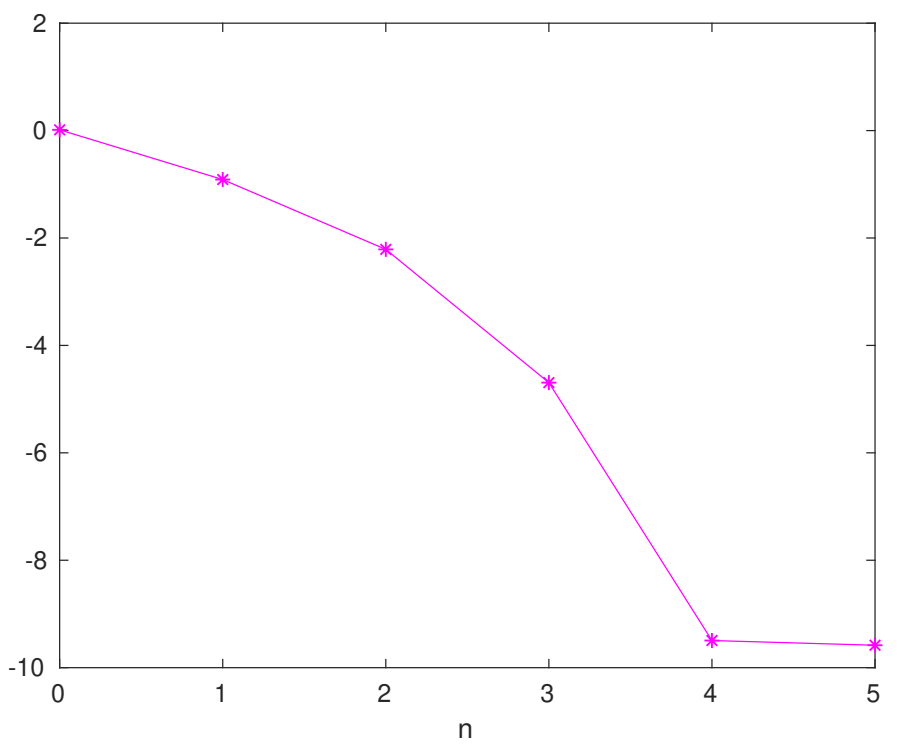

Figure 14: $\log _{10}\left(\left\|\mathbf{u}_{n+1}-\mathbf{u}_{n}\right\|_{\infty}\right)$ for case 6 .

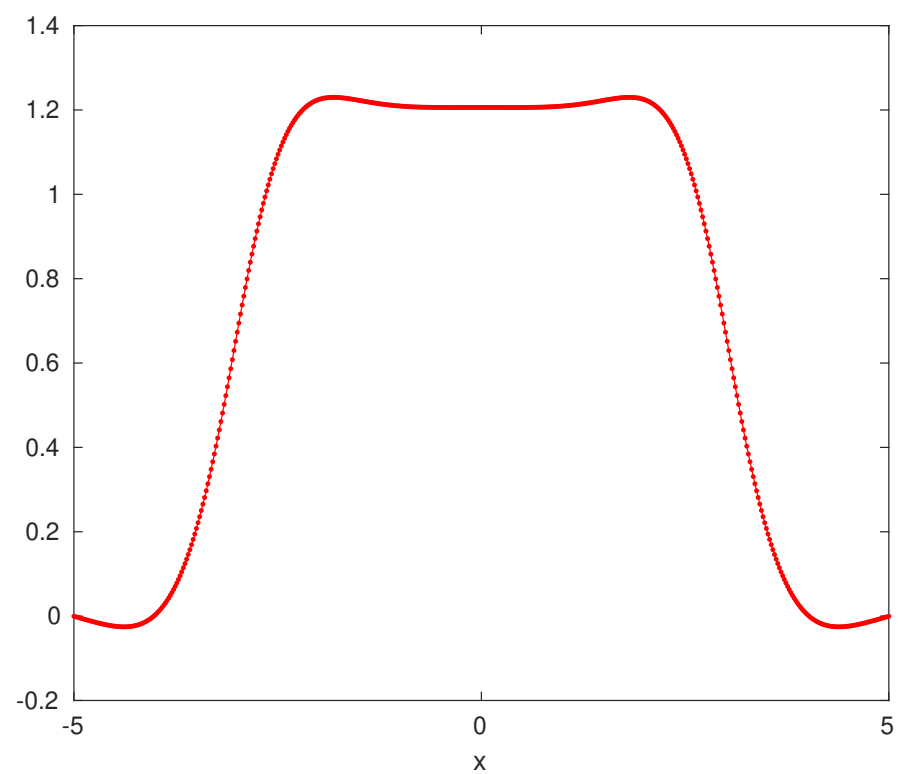

Figure 15: Solution for case 7 after 10 iterations 


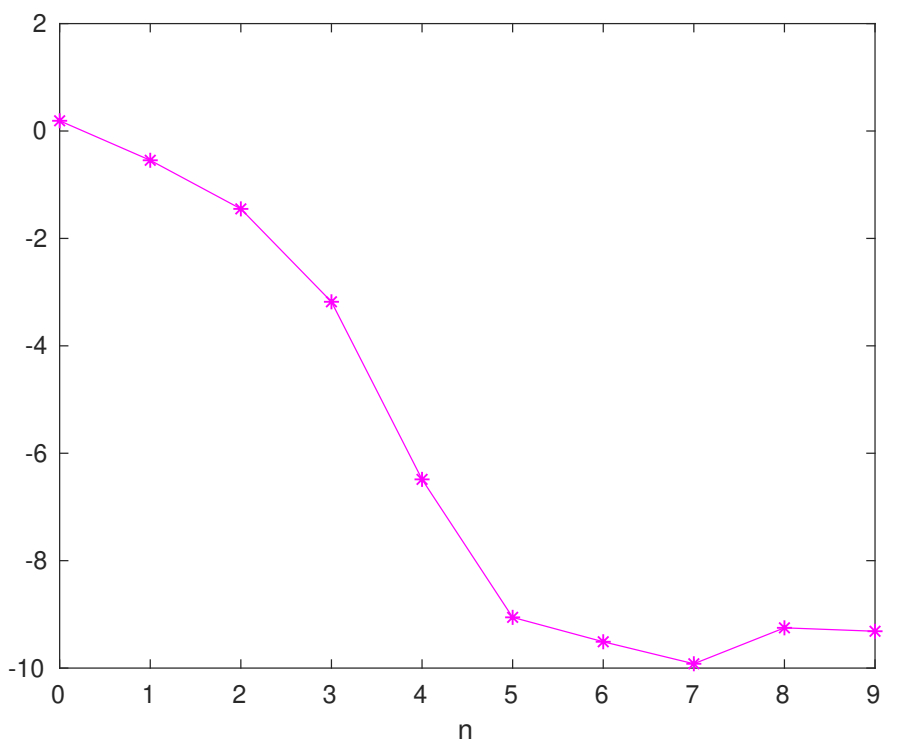

Figure 16: $\log _{10}\left(\left\|\mathbf{u}_{n+1}-\mathbf{u}_{n}\right\|_{\infty}\right)$ for case 7 .

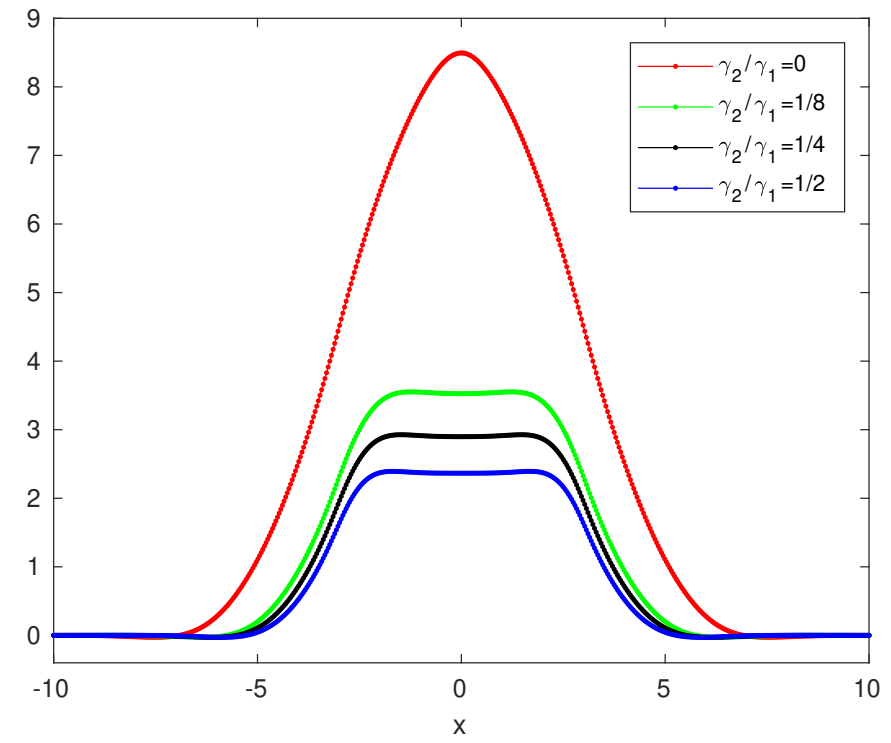

Figure 17: Solutions for case 8 after 10 iterations 


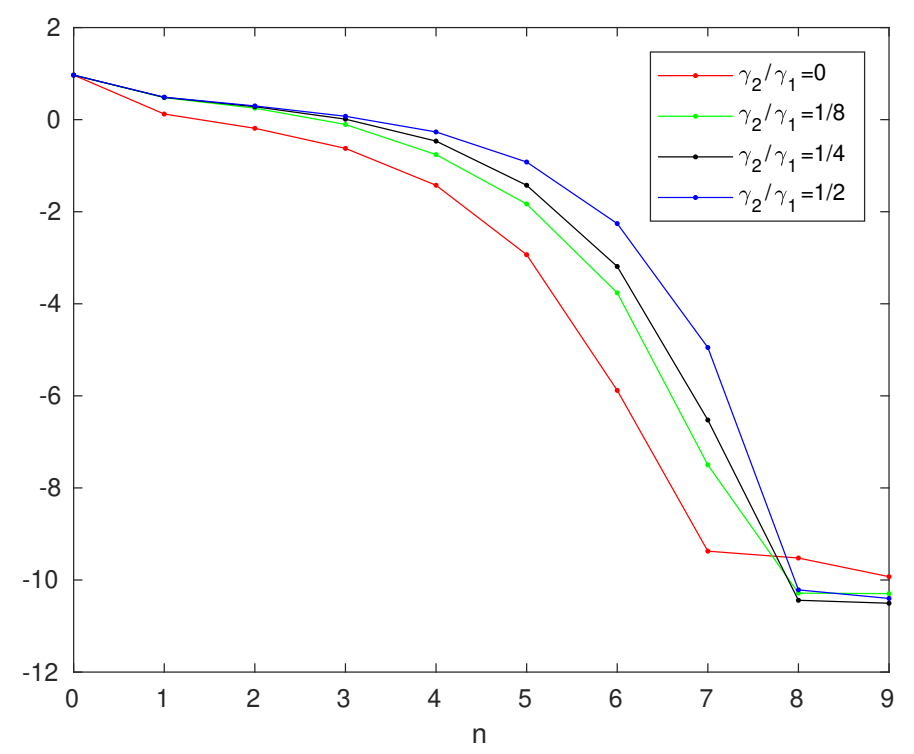

Figure 18: $\log _{10}\left(\left\|\mathbf{u}_{n+1}-\mathbf{u}_{n}\right\|_{\infty}\right)$ for case 8 .

number of iterations of the method ranges from 1 to 14 . Fig. 19 plots the computed solutions after 14 iterations. We can note that the solutions are very close to each other. Fig. 20 gives $\log _{10}\left(\left\|\mathbf{u}_{n+1}-\mathbf{u}_{n}\right\|_{\infty}\right)$ for $n$ ranging from 0 to 13 . Again, the plots are very close to each other. We can note that as $n$ increases $\left\|\mathbf{u}_{n+1}-\mathbf{u}_{n}\right\|_{\infty}$ is reduced significantly till reaching a value of the order of $10^{-11}$ for $n=13$, which is very small compared to the maximum absolute value of $u(x)$, which is $\approx 1.1$.

\section{Conclusions}

We have developed a method for solving numerically the differential equation governing the static deflections of an infinite nonlinear Bernouilli-Euler-von Karman beam with variable cross section resting on a nonlinear elastic foundation. The method combines quasilinearization with a new method for solving quasilinear differential equations involving up to the 4th order derivative based on discretizing the quasilinear differential equation by relating discretizations of up to the fourth order derivative of the solution with a discretization of the solution. Numerical experiments show that the error incurred by the discretization can be made small for the first, second and fourth derivatives and that the discretization method proposed in the paper converges fast and has good accuracy. Convergence of the method would only be guaranteed in the absence of discretization errors. For the nine cases taken as a benchmark for the method, convergence of the method till reaching optimal accuracy is achieved after no more than 14 iterations of quasilinearization. Regarding the achieved accuracy, it ranges from roughly four digits in relation to the largest absolute value of the solution to roughly eleven digits in relation to the largest absolute value of the solution. We believe that the optimal accuracy in the cases in which it is not very large can be improved by developing a new discretization method relating discretizations of up to the fourth order derivative with a discretization of the solution with better accuracy for the third derivative and we are currently pursuing that research direction.

\section{Acknowledgement}

The corresponding author (T. S. Jang, Prof.) was supported by Basic Research Program through the National Research Foundation of Korea (NKF) funded by the Ministry of Education (NRF-2015R1D1A1A01058542. 


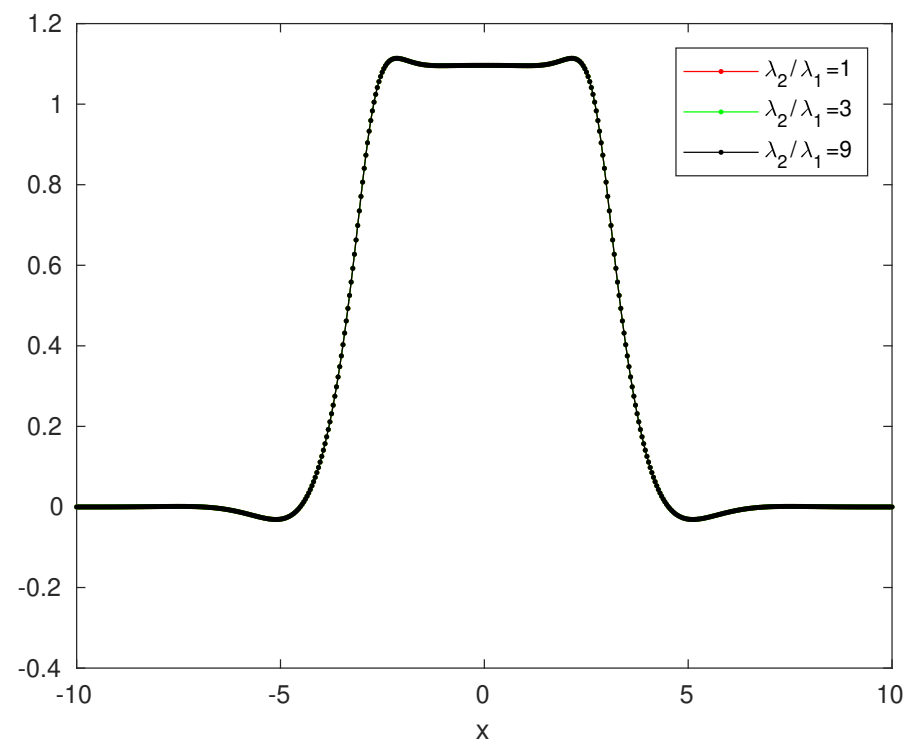

Figure 19: Solutions for case 9 after 13 iterations

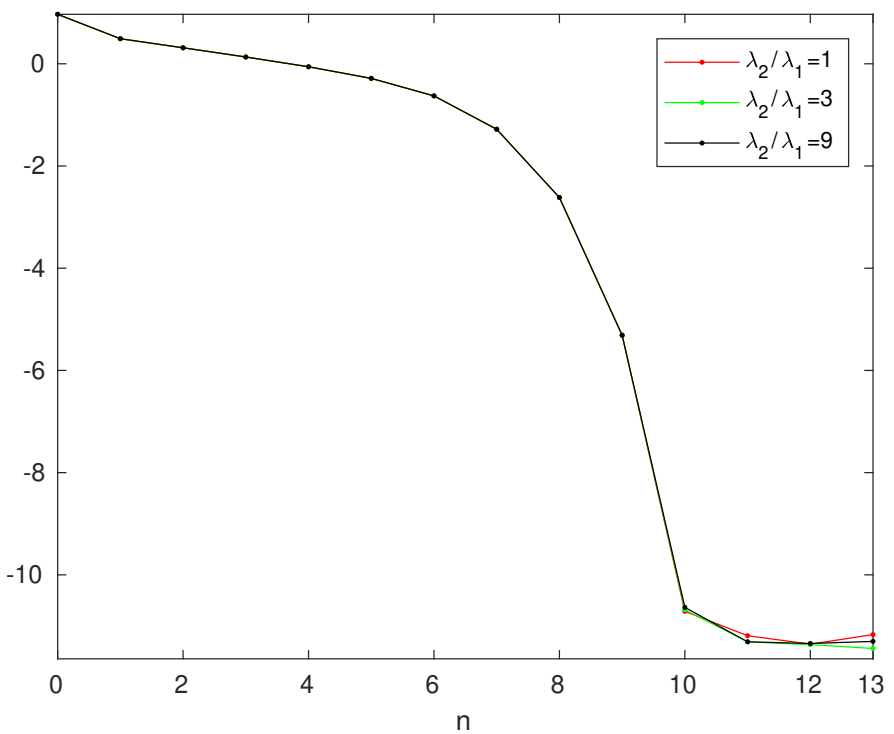

Figure 20: $\log _{10}\left(\left\|\mathbf{u}_{n+1}-\mathbf{u}_{n}\right\|_{\infty}\right)$ for case 9 . 


\section{Appendix}

Theorem. Let $G(x, y)$ be the Green function of a linear differential operator L. Let $\mathbf{G}$ be the matrix obtained by discretizing the Green function over an interval $[c, d]$ with a grid of points $x_{i}, 0 \leq i \leq n$ with $x_{0}=c$ and $x_{n}=d$. Then, the inverse of $\mathbf{G}$ exists.

Proof. By using the definition of the Green function we can write

$$
L G(x, y)=\delta(x-y),
$$

where $\delta(z)$ is the Dirac delta function. Let $f(x)$ be any continuous function. Using the previous equation we can write, for any $x \in[c, d]$,

$$
f(x)=\int_{c}^{d} \delta(x-y) f(y) d y=\int_{c}^{d} L G(x, y) f(y) d y .
$$

Let $\mathbf{L}$ be an approximate matrix representation of the linear differential operator $L$ in terms of values of the function to which the linear operator is applied at the grid points $x_{i}, 0 \leq i \leq n$. The corresponding vectorial representation of (13) is

$$
\left[\begin{array}{c}
f\left(x_{0}\right) \\
f\left(x_{1}\right) \\
\vdots \\
f\left(x_{n}\right)
\end{array}\right]=\int_{c}^{d} \mathbf{L}\left[\begin{array}{c}
G\left(x_{0}, y\right) \\
G\left(x_{1}, y\right) \\
\vdots \\
G\left(x_{n}, y\right)
\end{array}\right] f(y) d y
$$

Let $\mathbf{G}$ be the square matrix discretizing the Green function $G$ at the grid points, i.e. $G_{i, j}=G\left(x_{i}, x_{j}\right), 0 \leq i, j \leq n$. Using a quadrature rule in (14) based on averaging values of the function at the grid points $x_{i}, 0 \leq i \leq n$ with weights $b_{i}>0,0 \leq i \leq n$, where weight $b_{i}$ is associated with the value of the function at $x_{i}$, we get

$$
\left[\begin{array}{c}
f\left(x_{0}\right) \\
f\left(x_{1}\right) \\
\vdots \\
f\left(x_{n}\right)
\end{array}\right]=\mathbf{L}\left[\begin{array}{cccc}
G\left(x_{0}, x_{0}\right) & G\left(x_{0}, x_{1}\right) & \cdots & G\left(x_{0}, x_{n-1}\right) \\
G\left(x_{1}, x_{0}\right) & G\left(x_{1}, x_{1}\right) & \cdots & G\left(x_{1}, x_{n-1}\right) \\
\vdots & \vdots & \ddots & \vdots \\
G\left(x_{n}, x_{0}\right) & G\left(x_{n}, x_{1}\right) & \cdots & G\left(x_{n}, x_{n}\right)
\end{array}\right]\left[\begin{array}{c}
b_{0} f\left(x_{0}\right) \\
b_{1} f\left(x_{1}\right) \\
\vdots \\
b_{n} f\left(x_{n}\right)
\end{array}\right] .
$$

Then, denoting with $\mathbf{f}$ the vector

$$
\left[\begin{array}{c}
f\left(x_{0}\right) \\
f\left(x_{1}\right) \\
\vdots \\
f\left(x_{n}\right)
\end{array}\right]
$$

and with $\mathbf{b}$ the vector

$$
\left[\begin{array}{c}
b_{0} \\
b_{1} \\
\vdots \\
b_{n}
\end{array}\right]
$$

we have

$$
\mathbf{f}=\mathbf{L G} \operatorname{diag}[\mathbf{b}] \mathbf{f} .
$$

As $f$ is arbitrary, we must have

$$
\mathbf{I}=\mathbf{L G} \operatorname{diag}[\mathbf{b}],
$$

which implies that the inverse of $\mathbf{G d i a g}[\mathbf{b}]$ exists and, since the inverse of $\operatorname{diag}[\mathbf{b}]$ exists because $b_{j}>0,0 \leq j \leq n$, that the inverse of $\mathbf{G}$ exists. 


\section{References}

[1] S. Timoshenko, Method of analysis of statistical and dynamical stress in rails, Proceedings of international congress of applied mechanics. Zurich (1926), 407-418.

[2] D. Hui, Postbuckling behavior of infinite beams on elastic foundations using Koiter's improved theory. International Journal of Non-Linear Mechanics(1988), 23(2), 113-123.

[3] X. Ma, J.W. Butterworth, G.C. Clifton, Static analysis of an infinite beam resting on a tensionless Pasternak foundation, European Journal of Mechanics - A Solids (2009), 28(4), 697-703.

[4] Y.H. Kuo, S.Y. Lee, Deflection of non-uniform beams resting on a nonlinear elastic foundation, Computers and Structures (1994), 51, 513519.

[5] V. H. Nguyen, D. Duhamel, Finite element procedures for nonlinear structures in moving coordinates Part II: Infinite beam under moving harmonic loads, Computers and Structures (2008), 86, 2056-2063.

[6] T.S. Jang, H.S.Baek, J.K. Paik, A new method for the nonlinear deflection analysis of an infinite beam resting on a nonlinear elastic foundation, International Journal of Non-Linear Mechanics (2011), 46(1), 339-346.

[7] S.P. Sharma, S. Das Gupta, The bending problem of axially constrained beams on nonlinear elastic foundations, International Journal Solids and Structures (1975), 11(7-8), 853-859.

[8] E.J. Sapountzakis, A.E. Kampitsis, Nonlinear response of shear deformable beams on tensionless nonlinear viscoelastic foundation under moving loads, Journal of Sound and Vibration (2011), 330(22), 5410-5426.

[9] J.N. Reddy, An Introduction to Nonlinear finite element analysis, Oxford University Press, 2007.

[10] T.S. Jang, A new semi-analytical approach to large deflections of Bernoulli-Euler-von Karman beams on a linear elastic foundation: Nonlinear analysis of infinite beams, International Journal of Mechanical Sciences (2013), 66, 22-32.

[11] T.S. Jang and H.G. Sung, A new semi-analytical method for the non-linear static analysis of an infinite beam on a non-linear elastic foundation: a general approach to a variable beam cross-section, International Journal of Non-Linear Mechanics (2012), 47(4), 132-139.

[12] T.S. Jang, A general method for analyzing moderately large deflections of a non-uniform beam: An infinite Bernoulli-Euler-von Karman beam on a nonlinear elastic foundation. Acta Mechanica (2014), 225(7), 1967-1984

[13] R.E. Bellman, R.E. Kalaba, Quasilinearization and Nonlinear Boundary-Value Problems, Elsevier Publishing Company, New York, 1965.

[14] V.B. Mandelweig, Quasilinearization method and its verification on exactly solvable models in quantum mechanics, Journal of Mathematical Physics (1999) 40, 6266.

[15] R. Krivec, V.B. Mandelzweig, Numerical investigation of quasilinearization method in quantum mechanics, Computer Physics Communications, (2001), 138(1), 69-74.

[16] R. Kalaba, On nonlinear differential equations, the maximum operation and monotone convergence, Journal of Mathematics and Mechanics (1959), 8(4), 519-574.

[17] S.S. Motsa, P. Sibanda, Some modification of the quasilinearization method with higher-order convergence for solving nonlinear BVPs, Numerical algorithms (2013), 63(3), 399-417.

[18] E.S. Alaidarous, M.Z. Ullah, F. Ahmad, A.S. Al-Fhaid, An Efficient Higher-Order Quasilinearization Method for Solving Nonlinear BVPs, Journal of Applied Mathematics (2013), Article ID 258371.

[19] S. Cuomo, A. Marasco, A numerical approach to nonlinear two-point boundary value problems for ODEs, Computers and Mathematics with Applications (2008), 55(11), 2476-2489.

[20] Y.A. Brychkov, H.J. Glaeske, A.P. Prudnikov, V.K. Tuan, Multidimensional integral transformations, Gordon and Breach Science Publishers, 1992. 